East African Medical Journal Vol. 85 No. 10 October 2008

SENSITIVITY AND SPECIFICITY OF HIV RAPID TESTS USED FOR RESEARCH AND VOLUNTARY COUNSELLING AND TESTING

O. Anzala, MBChB, PhD, Associate Professor, Department of Medical Microbiology,College of Health Sciences, University of Nairobi and Programme Director, Kenya AIDS Vaccine Initiative, P. O. Box 19676 - 00200, Nairobi, Kenya, E. J. Sanders, MD, MPH, PhD, Principal Investigator, Kilifi Centre for Geographic Medicine Research Coast, Kilifi, Kenya, A. Kamali, Epidermiologist, MRC/UVRI Uganda Research Unit on AIDS, c/o Uganda Virus Research, P. O. Box 49, Entebbe, Uganda, M. Katende, MBChB, ADHSM, MPH,, Field Co ordinator, Joint Clinical Research Centre, Kakira Branch, Kampala, Uganda, G. N. Mutua, MBChB, MPH, Kenya AIDS Vaccine Initiative, P. O. Box 19676 - 00200, Nairobi, Kenya, E. Ruzagira, MBChB, MPH, MRC/UVRI Uganda Research Unit on AIDS, P. O. Box 49, Kampala, Uganda, G. Stevens, PhD, IAVI, South Africa, M. Simek BA, MPH, and M. Price, PhD, International AIDS Vaccine Initiative (IAVI)

Request for reprints to: Prof. O. Anzala, Department of Medical Microbiology, College of Health Sciences, University of Nairobi, P. O. Box 19676-00202, Nairobi, Kenya

\title{
SENSITIVITY AND SPECIFICITY OF HIV RAPID TESTS USED FOR RESEARCH AND VOLUNTARY COUNSELLING AND TESTING
}

\author{
O. ANZALA, E. J. SANDERS, A. KAMALI, M. KATENDE, G. N. MUTUA, \\ E. RUZAGIRA, G. STEVENS, M. SIMEK and M. PRICE
}

\begin{abstract}
Background: HIV rapid tests (RT) are a quick and non-technically demanding means to perform HIV voluntary counselling and testing (VCT) but understanding their limitations is vital to delivering quality VCT.

Objective: To determine the sensitivity and specificity of HIV rapid tests used for research and voluntary counselling and testing at four sites in East Africa.

Design: Cross-sectional study.

Setting: Masaka District, Uganda; a sugar plantation in Kakira, Uganda; Coastal Villages in the Kilifi District of Kenya; and the Urban slum of Kangemi located West of Nairobi, Kenya.

Subjects: Six thousands two hundred and fifty five consenting volunteers were enrolled into the study, and 675 prevalent HIV infections were identified.

Results: The RT sensitivity tended to be high for all assays at all sites $(97.63-100 \%)$ with the exception of the Uni-Gold assay $(90.24 \%$ in Kangemi, $96.58 \%$ in Kilifi). Twenty four RT results were recorded as 'weak positives', $22(92 \%)$ of which were negative by ELISA. There was a high rate of RT false positives in Uganda (positive predictive values ranging from $45.70 \%$ to $86.62 \%$ ).

Conclusions: The sensitivity and specificity of the RT varied significantly across sites. The rate of RT misclassification in Uganda suggests that a multiple test algorithm may be preferable to a single test as screener for HIV VCT.
\end{abstract}

\section{INTRODUCTION}

Traditional methods of HIV testing such as enzymelinked immunosorbent assays (ELISAs), and Western blots, which are currently used as the standard testing methods in the developed world, are often unavailable for resource-limited settings due to cost, turn around time to results, and technical limitations $(1,2)$. The use of rapid, less technically demanding tests to diagnose HIV infection has improved uptake of voluntary counselling and testing and facilitated HIV surveillance worldwide. Previous studies of HIV rapid tests (RT) have shown a similar sensitivity and specificity to traditional diagnostic methods (2-7). In this study, we tested a two assay HIV rapid algorithm, in four sites in Uganda and Kenya, to assess its reliability and validity in identifying HIV infection in individuals accessing voluntary counselling and testing (VCT) as part of HIV vaccine feasibility study.

\section{MATERIALS AND METHODS}

Study subjects: Male and female volunteers aged between 18 and 60 years willing to receive VCT for HIV and who provided informed consent to participate in an HIV vaccine feasibility study. The study involved a single visit to one of four sites: Rural villages in Masaka District, Uganda; a sugar plantation in Kakira, Uganda; Coastal Villages in 
the Kilifi District of Kenya; and the Urban slum of Kangemi located west of Nairobi, Kenya. This study was reviewed and approved by National Institutional Review Boards at each site.

Rapid tests: The Determine (Abbott Laboratories, Japan); Uni-Gold (Trinity Biotech, Ireland); and Capillus (Trinity Biotech) rapid test kits were used. Rapid test (RT) kits were used according to the manufacturer's instructions with one addition. When the Uni-Gold or Determine RT showed the patient test bar as present but fainter than the control bar, or if the Capillus assay showed signs of weak coagulation (there is no internal control for the Capillus rapid test), this was recorded as a "weak positive". As per the manufacturer's instructions, weak positives are considered a positive result for the purposes of this report; however, all weak positive results were confirmed by ELISA.
HIV testing algorithm: The HIV testing algorithm by site is shown in Figure I. Samples with discrepant RT results were subjected to confirmation by one or two ELISAs (Vironostika Uniform II, BioMerieux, Netherlands; and/or Murex HIV 1.2.0, Murex Biotech Ltd., UK), with Western blots (Genetic Systems, BioRad Laboratories) used to resolve any discrepancies in ELISA results for Masaka. In Kilifi, a third rapid test was used as a tiebreaker for discrepant RT results (lnstaScreen, GAIFAR) and ELISAs were not used. All specimens from volunteers diagnosed as HIV -infected were sent to an independent laboratory (Contract Laboratory Services, Johannesburg, South Africa) for confirmation of HIV antibody status by a third ELISA (Axsym HIV 1 and 2, Abbott Laboratories). In Masaka, all specimens found to be HIV antibody negative by RT were confirmed by dual ELISAs (Figure I), at the other sites, no further testing was done on volunteers diagnosed as HIV-uninfected.

Figure 1

HIV testing algorithms to identify infected individuals by site

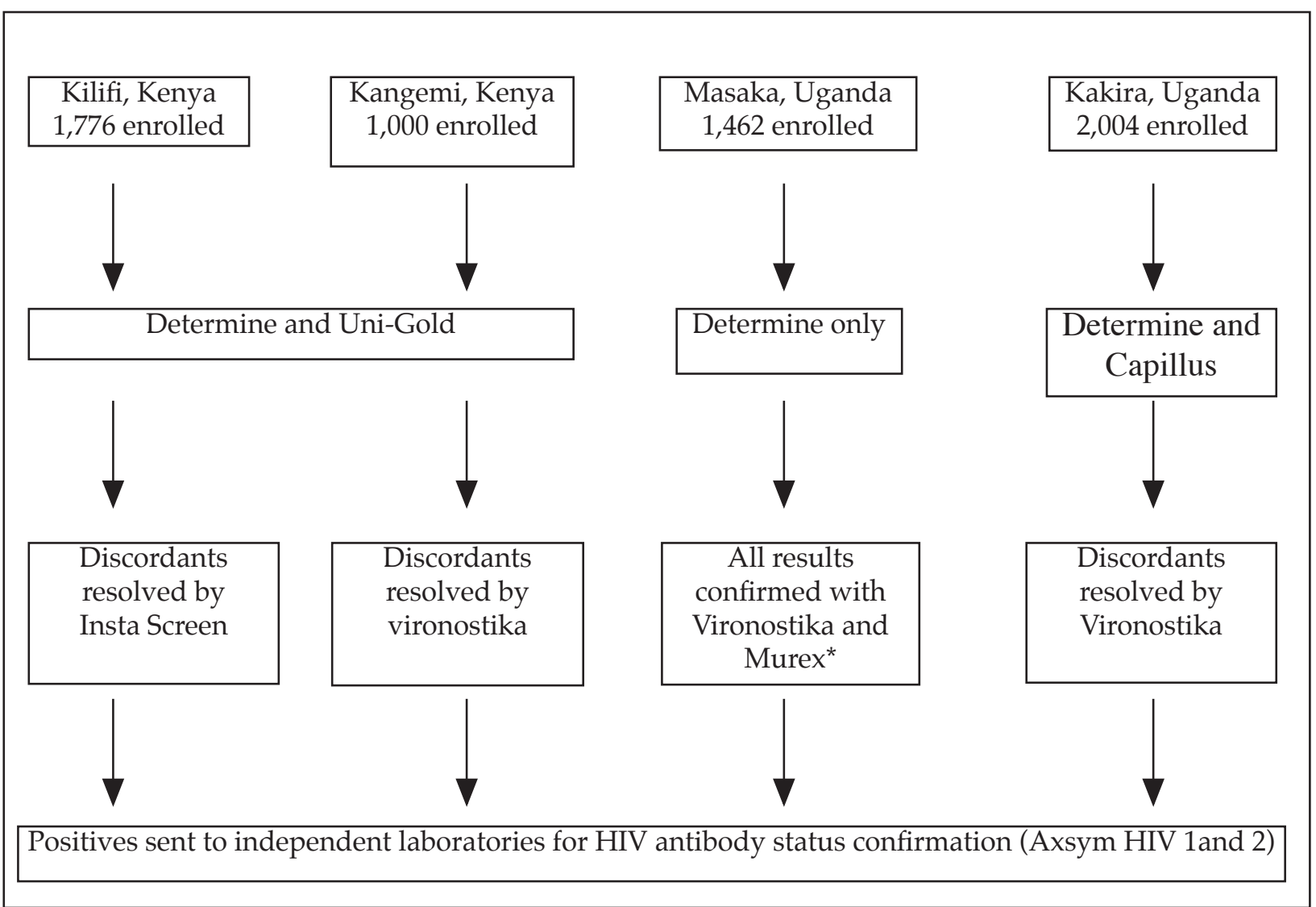

${ }^{*}$ Western Blot was used to resolve discrepant results in Masaka if ELISA results were discordant 
Assay accuracy: For each rapid test, the sensitivity, specificity, positive and negative predictive values, and overall accuracy were calculated. Sensitivity (the proportion of infected volunteers with antibody positive results) and specificity (the proportion of uninfected volunteers with antibody negative results) are shown as percentages. The Positive Predictive Value (PPV), the proportion of people with a positive RT or RT algorithm result who have antibody-positive HIV infection and Negative Predictive Value (NPV), the proportion of people with a negative RT or RT algorithm result who do not have antibody-positive HIV infection, shown as percentages, were calculated according to Bayes' theorem(8). The overall accuracy was calculated as the percentage of correct test results: $(a+d) /(a+b+c+d)$, where $a=$ true positives, $b=$ false positives, $\mathrm{c}=$ false negatives, and $\mathrm{d}=$ true negatives.

\section{RESULTS}

A total of 6,255 volunteers were enrolled into the study, and 675 prevalent infections were identified. For all sites the sensitivity and specificity of each rapid test varied (Table I).

The sensitivity, specificity, positive predictive value (PPV), negative predictive value (NPV) and accuracy of HIV rapid tests used at two sites in Kenya and two in Uganda
Twenty four positive results were recorded as weak positive (0 in Kangemi, 16 in Kakira, one in Masaka, and seven in Kilifi), 22 of which (92\%) were antibody negative by ELISA (i.e., FP). Of 131 volunteers with discrepant RT results (one test positive, one test negative), twenty six (19.8\%) volunteers were confirmed as HIV-infected by a third ELISA test. Specimens were not available for assessing viral load. This varied significantly by site, three cases out of $102(2.9 \%)$ in Kakira, $5 / 8$ (62.5\%) in Kilifi, and 18/21 (85.7\%) in Kangemi (Masaka used only one RT).

\section{DISCUSSION}

Overall, no rapid test performed well enough to recommend its use as a single diagnostic measurement. RT discrepant results were uncommon, and at Kangemi and Kilifi a high proportion of volunteers with these results were HIV -infected. Recent work at a STD clinic in Malawi reported that seven out of twenty one (33\%) of volunteers with PCRconfirmed acute HIV infection were RT discrepant (9). While only one Ugandan site used two RT, the majority of the HIV discrepant results came from this

\begin{tabular}{|c|c|c|c|c|c|c|c|}
\hline Site & $\begin{array}{l}\text { HIV } \\
\text { Prevalence }\end{array}$ & Assay(s) & $\begin{array}{c}\text { Sensitivity } \\
(\%)\end{array}$ & $\begin{array}{c}\text { Specificity } \\
(\%)\end{array}$ & $\begin{array}{c}\text { PPV } \\
0 / 0\end{array}$ & $\begin{array}{c}\text { NPV } \\
(\%)\end{array}$ & $\begin{array}{c}\text { Accuracy } \\
(\%)\end{array}$ \\
\hline Kangemi, & $164 / 1000$ & Determine & 98,78 & 99,76 & 97.38 & 99.89 & 99.60 \\
\hline Kenya & $(16.4 \%)$ & UniGold & 90.24 & 99.64 & 95.77 & 99.13 & 98.10 \\
\hline Kilifi, & $146 / 1769$ & Determine & 100 & 99.59 & 95.42 & 100 & 99.60 \\
\hline Kenya & $(8.3 \%)$ & UniGold & 96.58 & 99.83 & 98.60 & 99.69 & 99,60 \\
\hline Kakira, & $196 / 2004$ & Determine & 98.98 & 95.35 & 65.71 & 99.90 & 95.71 \\
\hline Uganda & $(9.8 \%)$ & Capillus & 99.49 & 98.62 & 86.62 & 99.95 & 98.70 \\
\hline $\begin{array}{l}\text { Masaka, } \\
\text { Uganda }\end{array}$ & $\begin{array}{l}169 / 1482 \\
(11.4 \%)\end{array}$ & Determine & 97.63 & 89.57 & 45.70 & 99.76 & 90.49 \\
\hline
\end{tabular}

The UniGold RT recorded the lowest sensitivities, at 90.24\% in Kangemi, and 96.58\% in Kilifi, however the UniGold specificity was high $(99.64 \%$ and 99.83\% respectively). The Determine RT, used at all sites, had a high sensitivity $(>97 \%)$, but the high rate of false positives (FP) recorded in Uganda resulted in low PPVs. The Capillus RT was used only in Kakira, performing with high sensitivity $(99.49 \%)$, however the number of FP observed with this assay resulted in a lower PPV (86.62\%). Due to the low number of FP in Kenya, the PPV of both rapid tests were high. site, and few (2.9\%) were truly HIV-infected. Although we found that the Determine assay performed with a high sensitivity at all four sites, its specificity at the Ugandan sites was lower, and the high rate of false positives resulted in low PPVs. Although the assay packageinserts for all three RT evaluated in this report do not mention weak positive results (the results are either positive or negative), the interpretation of these assays can be subjective, especially the Capillus assay which does not include an internal control. We observed $2 \%$ weak positive results, however more than $90 \%$ of these were FP. A similar experience has 
been reported by investigators in Rakai, Uganda (10). Although the investigators in this study reported a significant improvement in PPV by censoring their weak positive results, we found fewer weak positive results, and censoring them had negligible effects on the assay PPV (data not shown). The sensitivity and specificity of an HIV RT may vary depending on the local HIV strain and the population being tested (2, 11, 12). Problems with FP RT results have recently been reported in the US (13).

At three of the four sites, we did not confirm the antibody status of volunteers who were HIV negative by two rapid tests. While misdiagnosing infections as uninfected would affect both sensitivity and specificity, it is likely that these misclassifications are few; only five of $1,181(0.4 \%)$. Determine-negative results were confirmed as antibody-positive HIV infection in Masaka. The only site to confirm antibody status among all volunteers, Masaka, recorded the lowest sensitivity and specificity for the Determine assay. Originally, Masaka had begun this study with a two-RT algorithm, but due to a high rate of false positives noted early in the study we decided to switch to ELISA for confirmation of HIV status for all study volunteers. We did not have sufficient specimens to test for the presence of virus and it is possible that discrepant RT results confirmed as HIV uninfected by ELISA may have instead been due to early HIV infection prior to full seroconversion. A review of assay and laboratory procedures indicates that technician error or assay malfunction was unlikely, and it remains unclear what may have resulted in this poor specificity. Were we to use the most sensitive RT (Determine) as a screener to prompt confirmation by a second test, we would have missed 5/169 (3.0\%) infections in Masaka, and at least 2/164 (1.2\%) in Kangemi, 2/196 (1.0\%) in Kakira and 0/146 (0\%) in Kilifi. While a two-test algorithm is more expensive, the improvements in sensitivity, specificity, and predictive value are significant on the scale of national VCT programmes. With VCT uptake at government test sites nearly 800,000 in Kenya in 2006 (14) and 1.2 million in Uganda in 2004 (15), use of a single test algorithm could misdiagnose thousands of HIV infected individuals. The addition of a second test at a cost of approximately 1 US\$ per test would improve sensitivity at a cost of a few hundred dollars per HIV infected individual identified, making this algorithm improvement comparably cost-effective relative to other methods (16).

Our results suggest that a two-test algorithm can be utilised to rapidly identify HIV-infected individuals in East Africa, providing cost-effective improved diagnostic ability over a single RT used as a screening test. However, our results suggest that RT alone are not always effective; through RT testing prior to widespread use, and additional confirmatory diagnostic measures are highly recommended. As HIV continues to spread and access to anti-retroviral therapy expands throughout the developing world the ability to accurately and rapidly identify HIVinfected individuals will play a crucial role in preventing further transmission and providing life saving care and treatment $(11,12)$.

\section{ACKNOWLEDGEMENTS}

This work was made possible through funding from the International AIDS Vaccine Initiative. This paper is published with the permission of the Director, Kenya Medical Research Institute.

\section{REFERENCES}

I. Tamashiro, H., Maskill, W., Emmanuel, J., et al. Reducing the cost of HIV antibody testing. Lancet. 1993, 342: 87-90.

2. Lien, T.X., Tien, N.T.K., Chanpong G.F., et al. Evaluation of rapid diagnostic tests for the detection of human immunodeficiency virus types I and 2, hepatitis B surface antigen, and syphilis in Ho Chi Minh City, Vietnam. Am. J. Trop. Med. Hyg. 2000; 62: 301-309.

3. Bhore, A.V., Sastry, J, Patke, D., et al. Sensitivity and specificity of rapid HIV testing of pregnant women in India. Int. J. STD AIDS. 2003; 14: 37-41.

4. Koblavi-Deme, S., Maurice, C., Yavo, D., et al. Sensitivity and specificity of human immunodeficiency virus rapid serologic assays and testing algorithms in an antenatal clinic in Abidjan, Ivory Coast. J. Clin. Microbiol. 2001; 39: 1808-1812.

5. Palmer, C.J., Dubon, J.M., Koenig, E., et al. Field evaluation of the Determine rapid human immunodeficiency virus diagnostic test in Honduras and the Dominican Republic. J. Clin. Microbiol. 1999; 37: 3698-3700.

6. Salgado, C.D., Flanagan, H.L., Haverstick D.M., et al. Low rate of false-positive results with use of a rapid HIV test. Infect Control Hosp. Epidemiol. 2002; 23: 335-337.

7. van den Berk, G.E.L., Frissen, P.H.J., Regez, R.M., and P.J.G.M. Rietra, Evaluation of the rapid immunoassay determine HIV $1 / 2$ for detection of antibodies to human immunodeficiency virus types 1 and 2. J. Clin. Microbiol. 2003. 41: 3868-3869.

8. Fleiss, J., Levin, B., and Paik, C.M., Statistical Methods for Rates and Proportions. Wiley, 2003.

9. Fiscus, S.A., Pilcher, C.D., Miller, W. C., et al. Rapid, real-time detection of acute HIV infection in patients in Africa. J. Infect. Dis. 2007. 195: 416-424.

10. Gray, R.H., Makumbi, F., Serwadda, D., et al. Limitations of rapid HIV-1 tests during screening for trials in Uganda: diagnostic test accuracy study. Brit. Med. J. 2007; 335: 188.

11. Phillips, S., Granade, T.C., Chou-Pong, P., et al., Diagnosis of human immunodeficiency virus type 1 infection with different subtypes using rapid tests. Clin. Diagn. Lab. Immunol. 2000; 7: 698-699. 
12. Engelbrecht, S., G.J. de Jager, and van Rensburg, E.J. Evaluation of commercially available assays for antibodies to HIV -1 in serum obtained from South African patients infected with HIV-I subtypes B, C, and D. J. Med. Virol. 1994; 44: 223-228.

13. Wright, A, A. and Katz, I. T. Home testing for HIV. N. Engl. J. Med. 2006; 354: 437-440.

14. Kenyan National AIDS Control Programme, Kenya NASCOP National VCT Database - by December 2006,2007, Kenyan National AIDS Control Programme: Nairobi.
15. Uganda AIDS Commission, The Uganda HIV / AIDS Status Report, July 2004, December 2005, Uganda AIDS Commission: Kampala,

16. Walker, D. Cost and cost-effectiveness of HIV / AIDS prevention strategies in developing countries: Is there an evidence base? Health Policy Plan. 2003; 18: 4-17. 